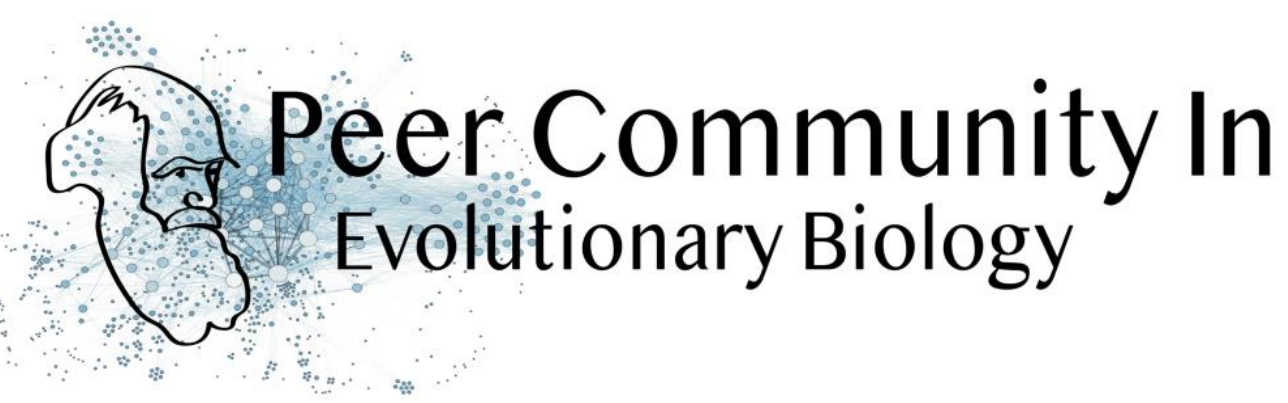

\title{
Y chromosome makes fruit flies die younger
}

\author{
Gabriel Marais, Jean-François Lemaitre and Cristina Vieira
}

A recommendation of:

Emily J Brown, Alison H Nguyen, Doris Bachtrog. The $Y$ chromosome may contribute to sex-specific ageing in Drosophila (2020). Nature ecology \& evolution: 4, 853-862. https://doi.org/10.1038/s41559-020-1179-5

$10.1101 / 156042$

Recommended: 29 July 2020

\section{Open Access}

Cite this recommendation as:

Gabriel Marais, Jean-François Lemaitre and Cristina Vieira (2020) Y chromosome makes fruit flies die younger. Peer Community in Evolutionary Biology, 100105. 10.24072/pci.evolbiol.100105

Published: 29 July 2020

Copyright: This work is licensed under the Creative Commons Attribution-NoDerivatives 4.0 International License. To view a copy of this license, visit http://creativecommons.org/licen ses/by-nd/4.0/
In most animal species, males and females display distinct survival prospect, a phenomenon known as sex gap in longevity (SGL, Marais et al. 2018). The study of SGLs is crucial not only for having a full picture of the causes underlying organisms' health, aging and death but also to initiate the development of sex-specific anti-aging interventions in humans (Austad and Bartke 2015). Three non-mutually evolutionary causes have been proposed to underlie SGLs (Marais et al.2018). First, SGLs could be the consequences of sex-differences in life history strategies. For example, evolving dimorphic traits (e.g. body size, ornaments or armaments) may imply unequal physiological costs (e.g. developmental, maintenance) between the sexes and this may result in differences in longevity and aging. Second, mitochondria are usually transmitted by the mother and thus selection is blind to mitochondrial deleterious mutations affecting only males. Such mutations can freely accumulate in the mitochondrial genome and may reduce male longevity, a phenomenon called the mother's curse (Frank and Hurst 1996). Third, in species with sex chromosomes, all recessive deleterious mutations will be expressed on the single $X$ chromosome in $X Y$ males and may reduce their longevity (the unguarded $X$ effect). In addition, the numerous transposable elements (TEs) on the $Y$ chromosome may affect aging. TE activity is normally repressed by epigenetic regulation (DNA methylation, histone modifications and small RNAs). However, it is known that this regulation is disrupted with increasing age. Because of the TE-rich $Y$ chromosome, more TEs may become active in old males than in old females, generating more somatic mutations, accelerating aging and reducing longevity in males (the toxic $Y$ effect, Marais et al. 2018).

The relative contributions of these different effects to SGLs remain unknown. Sexdifferences in life history strategies have been considered as the most important cause of SGLs for long (Tidière et al. 2015) but this effect remain equivocal (Lemaître et al. 2020) and cannot explain alone the diversity of patterns observed across species (Marais et al. 2018). Similarly, while studies in Drosophila and humans have shown that the 
mother's curse contributes to SGLs in those organisms (e.g. Milot et al. 2007), its contribution may not be strong. Recently, two large-scale comparative analyses have shown that in species with XY chromosomes males show a shorter lifespan compared to females, while in species with ZW chromosomes (a system in which the female are the heterogametic sex and are ZW, and the males ZZ) the opposite pattern is observed (Pipoly et al. 2015; Xirocostas et al. 2020). Apart from these correlational studies, very little experimental tests of the effect of sex chromosomes on longevity have been conducted. In Drosophila, the evidence suggests that the unguarded $X$ effect does not contribute to SGLs (Brengdahl et al. 2018). Whether a toxic $Y$ effect exists in this species was unknown.

In a very elegant study, Brown et al. (2020) provided strong evidence for such a toxic $\mathrm{Y}$ effect in Drosophila melanogaster. First, they checked that in the D. melanogaster strain that they were studying (Canton-S), males were indeed dying younger than females. They also confirmed that in this strain, as in others, the male genomes include more repeats and heterochromatin than the female ones using cytometry. A careful analysis of the heterochromatin (using $\mathrm{H} 3 \mathrm{~K} 9 \mathrm{me} 2$, a repressive histone modification typical of heterochromatin, as a proxy) in old flies revealed that heterochromatin loss was much more important in males than in females, in particular on the $Y$ chromosome (but also to a lesser extent at the pericentromric regions of the autosomes). This change in heterochromatin had two outputs, they found. First, the expression of the genes in those regions was affected. They highlighted that many of such genes are involved in immunity and regulation with a potential impact on longevity. Second, they found a striking TE reactivation. These two effects were stronger in males. While females showed clear reactivation of 6 TEs, with the total fraction of repeats in the transcriptome going from $2 \%$ (young females) to $4.6 \%$ (old females), males experienced the reactivation of $32 \mathrm{TEs}$, with the total fraction of repeats in the transcriptome going from $1.6 \%$ (young males) to $5.8 \%$ (old males). It appeared that most of these TEs are Y-linked. And when focusing on Y-linked repeats, they found that $32 \mathrm{Y}$-linked TEs became upregulated during male aging and the fraction of $Y$-linked TEs in the transcriptome increased ninefold.

All these observations clearly suggested that male longevity was decreased because of a toxic $Y$ effect. To really uncover a causal relationship between having a $Y$ chromosome and shorter longevity, Brown et al. (2020) artificially produced flies with atypical karyotypes: XO males, XXY females and XYY males. This is very interesting as they could uncouple the effect of the phenotypical sex (being male or female) and having a $Y$ chromosome or not, as in fruit flies sex is determined not by the $Y$ chromosome but by the $X /$ autosome ratio. Their results are striking. They found that longevity of the $X 0$ males was the highest (higher than $X X$ females in fact), and that of the XYY males the lowest. Females XXY had intermediate longevities. Importantly, this was found to be robust to genomic background as results were the same using crosses from different strains. When analysing TEs of these flies, they found a particularly strong expression of the $Y$-linked TEs in old XXY and XYY flies. Interestingly, in young XXY and XYY flies Y-linked TEs expression was also strong, suggesting the chromatin regulation of the $Y$ chromosome is disrupted in these flies.

This work points to the idea that SGLs in $D$. melanogaster are mainly explained by the toxic $Y$ effect. The molecular details however remain to be elucidated. The effect of the $Y$ chromosome on aging might be more complex than envisioned in the toxic $Y$ model presented above. Brown et al. (2020) indeed found that heterochromatin loss was globally faster in males, both at the $Y$ chromosome and the autosomes. The organisation of the nucleus, in particular of the nucleolus, which is involved in heterochromatin maintenance, involves the sex chromosomes in $D$. melanogaster as discussed in the paper, and may explain this observation. The epigenetic status of the $\mathrm{Y}$ chromosome is known to affect that of all the autosomes in Drosophila (Lemos et al. 2008). Also, in Brown et al. (2020) most of the work (in particular the genomic part) has been done on Canton-S. Only D. melanogaster was studied but limited data suggest different Drosophila species may have different SGLs. The TE analysis is known to be tricky, different tools to analyse TE expression exist (e.g. Lerat et al. 2017; Lanciano and Cristofari 2020). Future work should focus on testing the toxic $Y$ effect on other $D$. melanogaster strains and other Drosophila species, using different tools to study TE expression, and on dissecting the molecular details of the toxic $Y$ effect.

\section{References}

Austad, S. N., and Bartke, A. (2015). Sex differences in longevity and in responses to anti-aging interventions: A Mini-Review. Gerontology, 62(1), 40-46. https://doi.org/10.1159/000381472 


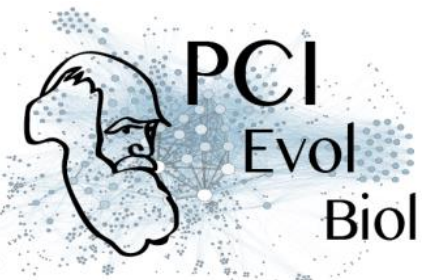

Brengdahl, M., Kimber, C. M., Maguire-Baxter, J., and Friberg, U. (2018). Sex differences in life span: Females homozygous for the $\mathrm{X}$ chromosome do not suffer the shorter life span predicted by the unguarded $\mathrm{X}$ hypothesis. Evolution; international journal of organic evolution, 72(3), 568-

577. https://doi.org/10.1111/evo.13434

Brown, E. J., Nguyen, A. H., and Bachtrog, D. (2020). The Y chromosome may contribute to sex-specific ageing in Drosophila. Nature ecology and evolution, 4(6), 853-862. https://doi.org/10.1038/s41559-020-1179-

5 or preprint link on bioRxiv

Frank, S. A., and Hurst, L. D. (1996). Mitochondria and male disease. Nature, 383(6597),

224. https://doi.org/10.1038/383224a0

Lanciano, S., and Cristofari, G. (2020). Measuring and interpreting transposable element expression. Nature reviews. Genetics, 10.1038/s41576-020-0251-y. Advance online publication. https://doi.org/10.1038/s41576020-0251-y

Lemaître, J. F., Ronget, V., Tidière, M., Allainé, D., Berger, V., Cohas, A., Colchero, F., Conde, D. A., Garratt, M., Liker, A., Marais, G., Scheuerlein, A., Székely, T., and Gaillard, J. M. (2020). Sex differences in adult lifespan and aging rates of mortality across wild mammals. Proceedings of the National Academy of Sciences of the United States of America, 117(15), 8546-8553. https://doi.org/10.1073/pnas.1911999117

Lemos, B., Araripe, L. O., and Hartl, D. L. (2008). Polymorphic Y chromosomes harbor cryptic variation with manifold functional consequences. Science (New York, N.Y.), 319(5859), 91-

93. https://doi.org/10.1126/science.1148861

Lerat, E., Fablet, M., Modolo, L., Lopez-Maestre, H., and Vieira, C. (2017). TEtools facilitates big data expression analysis of transposable elements and reveals an antagonism between their activity and that of piRNA genes. Nucleic acids research, 45(4), e17. https://doi.org/10.1093/nar/gkw953

Marais, G., Gaillard, J. M., Vieira, C., Plotton, I., Sanlaville, D., Gueyffier, F., and Lemaitre, J. F. (2018). Sex gap in aging and longevity: can sex chromosomes play a role?. Biology of sex differences, 9(1),

33. https://doi.org/10.1186/s13293-018-0181-y

Milot, E., Moreau, C., Gagnon, A., Cohen, A. A., Brais, B., and Labuda, D. (2017). Mother's curse neutralizes natural selection against a human genetic disease over three centuries. Nature ecology and evolution, 1(9), 1400-1406. https://doi.org/10.1038/s41559-017-0276-6

Pipoly, I., Bókony, V., Kirkpatrick, M., Donald, P. F., Székely, T., and Liker, A. (2015). The genetic sex-

determination system predicts adult sex ratios in tetrapods. Nature, 527(7576), 91-

94. https://doi.org/10.1038/nature15380

Tidière, M., Gaillard, J. M., Müller, D. W., Lackey, L. B., Gimenez, O., Clauss, M., and Lemaître, J. F. (2015). Does sexual selection shape sex differences in longevity and senescence patterns across vertebrates? A review and new insights from captive ruminants. Evolution; international journal of organic evolution, 69(12), 3123-3140. https://doi.org/10.1111/evo.12801

Xirocostas, Z. A., Everingham, S. E., and Moles, A. T. (2020). The sex with the reduced sex chromosome dies earlier: a comparison across the tree of life. Biology letters, 16(3),

20190867. https://doi.org/10.1098/rsbl.2019.0867 